\title{
Chebyshev Approximations for the Natural Logarithm of the Gamma Function*
}

\author{
By W. J. Cody and K. E. Hillstrom
}

Abstract. Rational Chebyshev approximations are given for the natural logarithm of the real gamma function for arguments in the intervals [0.5, 1.5], $[1.5,4.0]$ and $[4.0,12.0]$. Maximal relative errors range down to $1 \times 10^{-17}$.

1. Introduction. The computation of the gamma function for a real positive argument $x$ has been facilitated by the recent work of Rice [1], [2] giving rational Chebyshev approximations over the interval [2,3]. Rice has also generated approximations for $\ln \Gamma(x), x \geqq 12$, of the form

$$
\text { (1) } \ln \Gamma(x) \cong\left(x-\frac{1}{2}\right) \ln x-x+\ln (2 \pi)^{1 / 2}+(1 / x) P_{n}\left(1 / x^{2}\right) / Q_{m}\left(1 / x^{2}\right)
$$

where $P_{n}(y)$ and $Q_{m}(y)$ are polynomials of degree $n$ and $m$, respectively [2].

The computation of $\ln \Gamma(x)$ can thus be carried out directly from (1) for $x$ suffi(iently large. For smaller $x$ it is generally necessary to compute the gamma function first and then to take the natural logarithm. Compared to evaluating a rational function for $\ln \Gamma(x)$ directly, this process is fairly expensive. It is also frequently unstable numerically when $x$ is in the neighborhood of 1 or 2 , where the function vanishes.

This paper presents portions of the arrays of best rational approximations for $\ln \Gamma(x)$ on the intervals $[.5,1.5],[1.5,4.0]$, and $[4.0,12.0]$, thus complementing Rice's results. Rice has termed such arrays $I_{\infty}$ Walsh arrays.

2. The Approximation Forms. The approximation forms and intervals are:

$$
\begin{aligned}
& R_{n, m}^{0 *}(x)=-\ln x+R_{n, m}^{1 *}(x+1), \\
& 0<x \leqq 0.5 ; \\
& R_{n, m}^{1 *}(x)=(x-1) R_{n, m}^{1}(x), \\
& 0.5 \leqq x \leqq 1.5 ; \\
& R_{n, m}^{2 *}(x)=(x-2) R_{n, m}^{2}(x), \\
& 1.5 \leqq x \leqq 4.0 ;
\end{aligned}
$$

and

$$
R_{n, m}^{3 *}(x)=R_{n, m}^{3}(x),
$$$$
4.0 \leqq x \leqq 12.0 ;
$$

where here and in the following $R_{n, m}^{i}(x)=P_{n}{ }^{i}(x) / Q_{m}{ }^{i}(x)$, a ratio of polynomials. These forms correctly contain the logarithmic behaviour of the function as $x$ approaches zero from above, and the zeroes at $x=1$ and $x=2$. The particular partitioning of the interval [0.5, 12.0] was chosen because (i) reasonable accuracy is obtained in each subinterval for modest values of $n$ and $m$, and (ii) the approximations are somewhat balanced, i.e., the maximal errors in each subinterval for a given choice of $n, m$ are approximately the same.

Received February 21, 1966.

* Work performed under the auspices of the U. S. Atomic Energy Commission. 
3. Computations. The Remes algorithm for computing Chebyshev approximations [4] was used with 25 decimal floating point arithmetic on a CDC-3600 computer. The function $\ln \Gamma(x)$ was computed as needed using the well-known asymptotic form [3] and recurrence relation for most arguments, and the appropriate Taylor series expansions in the neighborhood of zeroes. The function values were verified to be correct to $20 \mathrm{~S}$ by using several thousand random arguments and comparing against alternate programs which had been checked against known values.

All error curves were levelled to at least $3 \mathrm{~S}$ in the maximal error. As a final check each approximation was separately tested for 2000 random arguments against

\section{TABLE I}

$$
E_{n, m}^{i *}=-100 \log \left\|\frac{\ln \Gamma(x)-R_{n, m}^{i *}(x)}{\ln \Gamma(x)}\right\|_{\infty}
$$

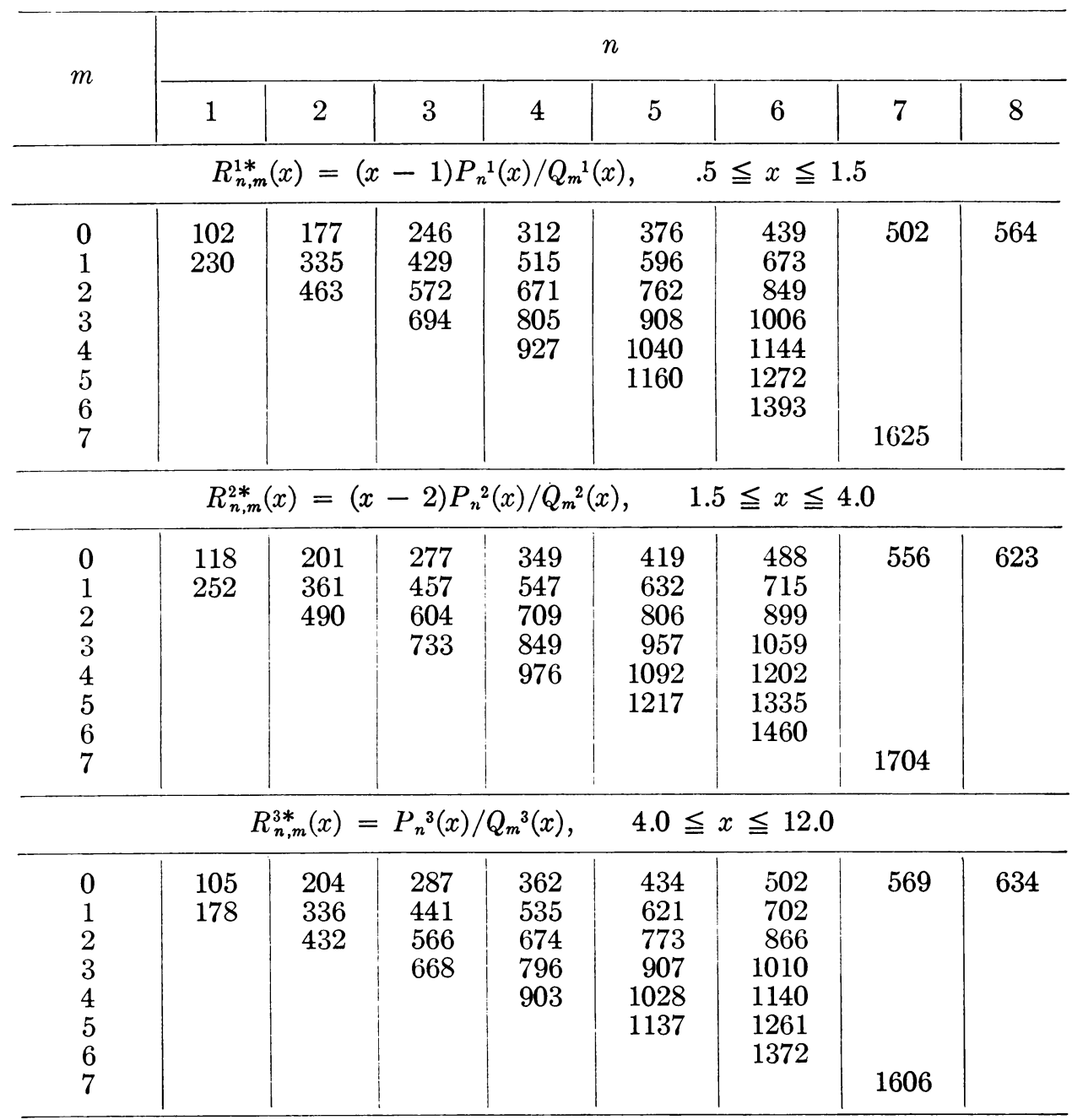


TABLE II

Coefficients for $R_{n, n}^{1 *}(x)=(x-1) \sum_{i=0}^{n} P_{i}{ }^{1} x^{i} / \sum_{i=0}^{n} Q_{i}{ }^{1} x^{i}, \quad .5 \leqq x \leqq 1.5$

\begin{tabular}{|c|c|c|c|c|}
\hline$i$ & \multicolumn{2}{|l|}{$P_{i}{ }^{1}$} & \multicolumn{2}{|l|}{$Q_{i}{ }^{1}$} \\
\hline \multicolumn{5}{|c|}{$n=1$} \\
\hline $\begin{array}{l}0 \\
1\end{array}$ & $\begin{array}{r}-2.2105 \\
1.0740\end{array}$ & $\begin{array}{l}(00) \\
(00)\end{array}$ & $\begin{array}{l}9.6932 \\
1.0000\end{array}$ & $\begin{array}{r}(-01) \\
(00)\end{array}$ \\
\hline \multicolumn{5}{|c|}{$n=2$} \\
\hline $\begin{array}{l}0 \\
1 \\
2\end{array}$ & $\begin{array}{r}-2.607538 \\
-2.743069 \\
2.021198\end{array}$ & $\begin{array}{l}(00) \\
(00) \\
(00)\end{array}$ & $\begin{array}{l}8.013237 \\
3.966653 \\
1.000000\end{array}$ & $\begin{array}{r}(-01) \\
(00) \\
(00)\end{array}$ \\
\hline \multicolumn{5}{|c|}{$n=3$} \\
\hline $\begin{array}{l}0 \\
1 \\
2 \\
3\end{array}$ & 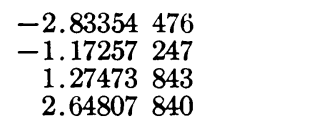 & $\begin{array}{l}(00) \\
(01) \\
(00) \\
(00)\end{array}$ & $\begin{array}{ll}7.26957 & 927 \\
8.00937 & 488 \\
8.69084 & 166 \\
1.00000 & 000\end{array}$ & $\begin{array}{r}(-01) \\
(00) \\
(00) \\
(00)\end{array}$ \\
\hline \multicolumn{5}{|c|}{$n=4$} \\
\hline $\begin{array}{l}0 \\
1 \\
2 \\
3 \\
4\end{array}$ & 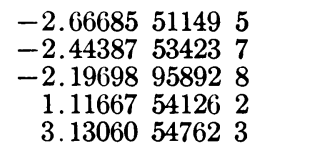 & $\begin{array}{l}(00) \\
(01) \\
(01) \\
(01) \\
(00)\end{array}$ & $\begin{array}{lll}6.07771 & 38777 & 1 \\
1.19400 & 90572 & 1 \\
3.14690 & 11574 & 9 \\
1.52346 & 87407 & 0 \\
1.00000 & 00000 & 0\end{array}$ & $\begin{array}{r}(-01) \\
(01) \\
(01) \\
(01) \\
(00)\end{array}$ \\
\hline \multicolumn{5}{|c|}{$n=5$} \\
\hline $\begin{array}{l}0 \\
1 \\
2 \\
3 \\
4 \\
5\end{array}$ & $\begin{array}{rrr}-2.60940 & 66054 & 623 \\
-4.15090 & 18875 & 434 \\
-9.55641 & 17677 & 317 \\
-1.18114 & 39967 & 596 \\
2.81137 & 44347 & 038 \\
3.51735 & 89912 & 443\end{array}$ & $\begin{array}{l}(00) \\
(01) \\
(01) \\
(01) \\
(01) \\
(00)\end{array}$ & $\begin{array}{lll}5.45875 & 04274 & 950 \\
1.66749 & 69701 & 154 \\
7.85560 & 98036 & 754 \\
8.72893 & 05773 & 548 \\
2.35907 & 62639 & 739 \\
1.00000 & 00000 & 000\end{array}$ & $\begin{array}{r}(-01) \\
(01) \\
(01) \\
(01) \\
(01) \\
(00)\end{array}$ \\
\hline \multicolumn{5}{|c|}{$n=6$} \\
\hline $\begin{array}{l}0 \\
1 \\
2 \\
3 \\
4 \\
5 \\
6\end{array}$ & $\begin{array}{rrr}-2.33590 & 98949 & 51284 \\
-5.75008 & 93603 & 04123 \\
-2.45872 & 61722 & 29242 \\
-2.15135 & 13573 & 72570 \\
5.55840 & 45723 & 51531 \\
5.27068 & 93753 & 00983 \\
3.84287 & 36567 & 45991\end{array}$ & $\begin{array}{l}(00) \\
(01) \\
(02) \\
(02) \\
(01) \\
(01) \\
(00)\end{array}$ & $\begin{array}{lll}4.57174 & 20282 & 50299 \\
2.01068 & 51344 & 33395 \\
1.50068 & 39064 & 89095 \\
3.08829 & 54973 & 42428 \\
1.93877 & 84034 & 37713 \\
3.37330 & 47907 & 07074 \\
1.00000 & 00000 & 00000\end{array}$ & $\begin{array}{r}(-01) \\
(01) \\
(02) \\
(02) \\
(02) \\
(01) \\
(00)\end{array}$ \\
\hline
\end{tabular}

$n=7$

\begin{tabular}{|c|c|c|c|c|}
\hline & $\begin{array}{rrrr}-2.20884 & 39972 & 16182 & 306 \\
-7.74106 & 40713 & 32953 & 034 \\
-5.17638 & 34980 & 23217 & 924 \\
-9.22261 & 37288 & 01521 & 582 \\
-2.61721 & 85838 & 56145 & 190 \\
2.43175 & 24352 & 44210 & 223 \\
8.56898 & 20628 & 31317 & 339 \\
4.12084 & 31858 & 47770 & 031\end{array}$ & $\begin{array}{l}(00) \\
(01) \\
(02) \\
(02) \\
(02) \\
(02) \\
(01) \\
(00)\end{array}$ & $\begin{array}{llll}4.09779 & 29210 & 92615 & 065 \\
2.44351 & 96625 & 06311 & 704 \\
2.62308 & 34702 & 69460 & 180 \\
8.46075 & 53620 & 20782 & 006 \\
9.51323 & 59767 & 97059 & 772 \\
3.77837 & 24848 & 23942 & 081 \\
4.56467 & 71875 & 85907 & 957 \\
1.00000 & 00000 & 00000 & 000\end{array}$ & $\begin{array}{r}(-01) \\
(01) \\
(02) \\
(02) \\
(02) \\
(02) \\
(01) \\
(00)\end{array}$ \\
\hline
\end{tabular}


Coefficients for $R_{n \cdot n}^{2 *}(x)=(x-2) \sum_{i=0}^{n} P_{i}{ }^{2} x^{i} / \sum_{i=0}^{n} Q_{i}{ }^{2} x^{i}, \quad 1.5 \leqq x \leqq 4.0$

\begin{tabular}{|c|c|c|c|c|}
\hline$i$ & \multicolumn{2}{|l|}{$P_{i}{ }^{2}$} & \multicolumn{2}{|l|}{$Q_{i^{2}}$} \\
\hline \multicolumn{5}{|c|}{$n=1$} \\
\hline $\begin{array}{l}0 \\
1\end{array}$ & $\begin{array}{r}-2.0456 \\
2.1327\end{array}$ & $\begin{array}{l}(00) \\
(00)\end{array}$ & $\begin{array}{l}3.2610 \\
1.0000\end{array}$ & $\begin{array}{l}(00) \\
(00)\end{array}$ \\
\hline \multicolumn{5}{|c|}{$n=2$} \\
\hline $\begin{array}{l}0 \\
1 \\
2\end{array}$ & $\begin{array}{r}-8.506018 \\
5.482955 \\
3.042595\end{array}$ & $\begin{array}{l}(00) \\
(00) \\
(00)\end{array}$ & $\begin{array}{l}7.881893 \\
1.136112 \\
1.000000\end{array}$ & $\begin{array}{l}(00) \\
(01) \\
(00)\end{array}$ \\
\hline \multicolumn{5}{|c|}{$n=3$} \\
\hline $\begin{array}{l}0 \\
1 \\
2 \\
3\end{array}$ & $\begin{array}{rr}-2.62608 & 1587 \\
-8.39906 & 1479 \\
3.09835 & 8770 \\
3.68073 & 1176\end{array}$ & $\begin{array}{l}(01) \\
(00) \\
(01) \\
(00)\end{array}$ & $\begin{array}{ll}1.86074 & 2491 \\
6.76133 & 4331 \\
2.47764 & 2509 \\
1.00000 & 0000\end{array}$ & $\begin{array}{l}(01) \\
(01) \\
(01) \\
(00)\end{array}$ \\
\hline \multicolumn{5}{|c|}{$n=4$} \\
\hline $\begin{array}{l}0 \\
1 \\
2 \\
3 \\
4\end{array}$ & $\begin{array}{r}-7.83359299449 \\
-1.42046296688 \\
1.37519416416 \\
7.86994924154 \\
4.16438922228\end{array}$ & $\begin{array}{l}(01) \\
(02) \\
(02) \\
(01) \\
(00)\end{array}$ & $\begin{array}{lll}4.70668 & 76606 & 0 \\
3.13399 & 21589 & 4 \\
2.63505 & 07472 & 1 \\
4.33400 & 02251 & 4 \\
1.00000 & 00000 & 0\end{array}$ & $\begin{array}{l}(01) \\
(02) \\
(02) \\
(01) \\
(00)\end{array}$ \\
\hline \multicolumn{5}{|c|}{$n=5$} \\
\hline $\begin{array}{l}0 \\
1 \\
2 \\
3 \\
4 \\
5\end{array}$ & $\begin{array}{rrr}-2.16192 & 29262 & 4703 \\
-8.27790 & 89780 & 9598 \\
1.82987 & 82201 & 2009 \\
7.06543 & 70015 & 4966 \\
1.49903 & 66270 & 9861 \\
4.54827 & 47772 & 3909\end{array}$ & $\begin{array}{l}(02) \\
(02) \\
(02) \\
(02) \\
(02) \\
(00)\end{array}$ & $\begin{array}{lll}1.16412 & 65946 & 1333 \\
1.20459 & 29366 & 3292 \\
1.85645 & 03568 & 6087 \\
7.05287 & 06971 & 5149 \\
6.65573 & 50746 & 7416 \\
1.00000 & 00000 & 0000\end{array}$ & $\begin{array}{l}(02) \\
(03) \\
(03) \\
(02) \\
(01) \\
(00)\end{array}$ \\
\hline
\end{tabular}

$n=6$

\begin{tabular}{|c|c|c|c|c|}
\hline $\begin{array}{l}0 \\
1 \\
2 \\
3 \\
4 \\
5 \\
6\end{array}$ & $\begin{array}{rrrr}-5.60177 & 73537 & 80387 & 7 \\
-3.69298 & 34005 & 59128 & 2 \\
-1.97780 & 70769 & 84164 & 6 \\
3.79751 & 24011 & 52511 & 8 \\
2.17973 & 66058 & 89591 & 5 \\
2.48845 & 25168 & 57407 & 6 \\
4.87402 & 01396 & 83863 & 6\end{array}$ & $\begin{array}{l}(02) \\
(03) \\
(03) \\
(03) \\
(03) \\
(02) \\
(00)\end{array}$ & $\begin{array}{llll}2.76785 & 83623 & 80410 & 1 \\
4.16994 & 15153 & 20023 & 1 \\
1.04595 & 76594 & 05895 & 9 \\
7.23400 & 87928 & 94807 & 1 \\
1.56120 & 45277 & 92863 & 5 \\
9.50999 & 17418 & 20893 & 8 \\
1.00000 & 00000 & 00000 & 0\end{array}$ & $\begin{array}{l}(02) \\
(03) \\
(04) \\
(03) \\
(03) \\
(01) \\
(00)\end{array}$ \\
\hline \multicolumn{5}{|c|}{$n=7$} \\
\hline $\begin{array}{l}0 \\
1 \\
2 \\
3 \\
4 \\
5 \\
6 \\
7\end{array}$ & $\begin{array}{rrrr}-1.51383 & 18341 & 15066 & 7785 \\
-1.50863 & 02287 & 66725 & 0272 \\
-2.06482 & 94205 & 32528 & 3281 \\
1.20431 & 73809 & 87164 & 0151 \\
1.95536 & 05540 & 63044 & 9846 \\
5.26898 & 32559 & 14981 & 2458 \\
3.77510 & 67979 & 72170 & 2241 \\
5.15505 & 76176 & 40817 & 1704\end{array}$ & $\begin{array}{l}(03) \\
(04) \\
(04) \\
(04) \\
(04) \\
(03) \\
(02) \\
(00)\end{array}$ & $\begin{array}{llll}6.98327 & 41405 & 73510 & 2159 \\
1.44020 & 90371 & 70085 & 2304 \\
5.26228 & 63838 & 41199 & 2470 \\
5.71202 & 55396 & 02502 & 9854 \\
2.20295 & 62144 & 15663 & 6889 \\
3.03990 & 30414 & 39439 & 8824 \\
1.28909 & 31890 & 12957 & 6873 \\
1.00000 & 00000 & 00000 & 0000\end{array}$ & $\begin{array}{l}(02) \\
(04) \\
(04) \\
(04) \\
(04) \\
(03) \\
(02) \\
(00)\end{array}$ \\
\hline
\end{tabular}


TABLE IV

Coefficients for $R_{n, n}^{3^{*}}(x)=\sum_{i=0}^{n} P_{i^{3} x^{i}} / \sum_{i=0}^{n} Q_{i}{ }^{3} x^{i}, \quad 4.0 \leqq x \leqq 12.0$

\begin{tabular}{|c|c|c|c|c|}
\hline$i$ & \multicolumn{2}{|l|}{$P_{\boldsymbol{i}^{3}}$} & \multicolumn{2}{|l|}{$Q i^{3}$} \\
\hline \multicolumn{5}{|c|}{$n=1$} \\
\hline $\begin{array}{l}0 \\
1\end{array}$ & $\begin{array}{r}9.910 \\
-3.677\end{array}$ & $\begin{array}{l}(01) \\
(01)\end{array}$ & $\begin{array}{r}-3.122 \\
1.000\end{array}$ & $\begin{array}{l}(01) \\
(00)\end{array}$ \\
\hline \multicolumn{5}{|c|}{$n=2$} \\
\hline $\begin{array}{l}0 \\
1 \\
2\end{array}$ & $\begin{array}{r}-1.637744 \\
7.748569 \\
-3.324363\end{array}$ & $\begin{array}{l}(02) \\
(02) \\
(02)\end{array}$ & $\begin{array}{r}-8.407843 \\
-1.263600 \\
1.000000\end{array}$ & $\begin{array}{l}(02) \\
(02) \\
(00)\end{array}$ \\
\hline \multicolumn{5}{|c|}{$n=3$} \\
\hline $\begin{array}{l}0 \\
1 \\
2 \\
3\end{array}$ & $\begin{array}{r}-1.37219936 \\
2.05137737 \\
-4.71572823 \\
-1.04210703\end{array}$ & $\begin{array}{l}(04) \\
(04) \\
(03) \\
(03)\end{array}$ & $\begin{array}{r}-1.13338846 \\
-6.27235378 \\
-3.02297001 \\
1.00000000\end{array}$ & $\begin{array}{l}(04) \\
(03) \\
(02) \\
(00)\end{array}$ \\
\hline \multicolumn{5}{|c|}{$n=4$} \\
\hline $\begin{array}{l}0 \\
1 \\
2 \\
3 \\
4\end{array}$ & $\begin{array}{rrr}-2.1215957232 & 3 \\
2.30661 & 51061 & 6 \\
2.74647 & 64470 & 5 \\
-4.02621 & 11997 & 5 \\
-2.29660 & 72978 & 0\end{array}$ & $\begin{array}{l}(05) \\
(05) \\
(04) \\
(04) \\
(03)\end{array}$ & $\begin{array}{r}-1.16328495004 \\
-1.46025937511 \\
-2.42357409629 \\
-5.70691009324 \\
1.00000000000\end{array}$ & $\begin{array}{l}(05) \\
(05) \\
(04) \\
(02) \\
(00)\end{array}$ \\
\hline \multicolumn{5}{|c|}{$n=5$} \\
\hline $\begin{array}{l}0 \\
1 \\
2 \\
3 \\
4 \\
5\end{array}$ & $\begin{array}{rrr}-2.42731 & 13085 & 758 \\
1.38608 & 69828 & 508 \\
1.85377 & 73351 & 564 \\
-6.42799 & 27530 & 351 \\
-1.55159 & 71577 & 126 \\
-4.21052 & 09252 & 847\end{array}$ & $\begin{array}{l}(06) \\
(06) \\
(06) \\
(05) \\
(05) \\
(03)\end{array}$ & $\begin{array}{rrr}-1.05424 & 82321 & 634 \\
-2.45157 & 05199 & 457 \\
-8.62741 & 86723 & 037 \\
-6.77712 & 58633 & 073 \\
-9.41366 & 13234 & 388 \\
1.00000 & 00000 & 000\end{array}$ & $\begin{array}{l}(06) \\
(06) \\
(05) \\
(04) \\
(02) \\
(00)\end{array}$ \\
\hline \multicolumn{5}{|c|}{$n=6$} \\
\hline $\begin{array}{l}0 \\
1 \\
2 \\
3 \\
4 \\
5 \\
6\end{array}$ & $\begin{array}{rrrr}-2.48043 & 69488 & 28593 \\
-3.35677 & 82814 & 54576 \\
3.63218 & 04931 & 54257 \\
-2.94234 & 45930 & 32234 \\
-4.75045 & 94653 & 43956 \\
-4.30699 & 69819 & 57098 \\
-6.88062 & 40094 & 59425\end{array}$ & $\begin{array}{l}(07) \\
(06) \\
(07) \\
(06) \\
(06) \\
(05) \\
(03)\end{array}$ & $\begin{array}{rrr}-9.16055 & 82863 & 71317 \\
-3.45441 & 75093 & 34395 \\
-2.09696 & 23255 & 80444 \\
-3.41525 & 17108 & 01107 \\
-1.55528 & 90280 & 85353 \\
-1.42168 & 29839 & 65146 \\
1.00000 & 00000 & 00000\end{array}$ & $\begin{array}{l}(06) \\
(07) \\
(07) \\
(06) \\
(05) \\
(03) \\
(00)\end{array}$ \\
\hline \multicolumn{5}{|c|}{$n=7$} \\
\hline $\begin{array}{l}0 \\
1 \\
2 \\
3 \\
4 \\
5 \\
6 \\
7\end{array}$ & $\begin{array}{rrrr}-2.40798 & 69801 & 73375 & 493 \\
-2.44832 & 17690 & 32881 & 564 \\
4.81807 & 71027 & 73628 & 010 \\
1.11938 & 53542 & 99855 & 449 \\
-8.73167 & 54382 & 38386 & 656 \\
-1.97183 & 01158 & 60920 & 573 \\
-9.82710 & 22814 & 20492 & 083 \\
-1.03770 & 16517 & 32974 & 263\end{array}$ & $\begin{array}{l}(08) \\
(08) \\
(08) \\
(08) \\
(07) \\
(07) \\
(05) \\
(04)\end{array}$ & $\begin{array}{rrrr}-7.90261 & 11141 & 87634 & 109 \\
-4.35370 & 71480 & 43741 & 914 \\
-4.04435 & 92829 & 14355 & 059 \\
-1.11925 & 41162 & 63318 & 226 \\
-1.04857 & 75830 & 49937 & 280 \\
-3.11406 & 28473 & 40678 & 552 \\
-2.01527 & 51955 & 00482 & 591 \\
1.00000 & 00000 & 00000 & 000\end{array}$ & $\begin{array}{l}(07) \\
(08) \\
(08) \\
(08) \\
(07) \\
(05) \\
(03) \\
(00)\end{array}$ \\
\hline
\end{tabular}


the original function routines. In all cases maximal errors agreed, within roundoff, in magnitude and position with those given by the error curves in the Remes algorithm.

4. Results. Table I lists the values of

$$
E_{n, m}^{i *}=-100 \log \left\|\frac{\ln \Gamma(x)-R_{n, m}^{i *}(x)}{\ln \Gamma(x)}\right\|_{\infty}
$$

for the initial segments of the $L_{\infty}$ Walsh arrays. An examination of the tables indicates $E_{n, m}^{i *}$ is maximal for fixed $n+m$ along the line $n=m$ for the first two intervals, and is not far from maximal along this line in the last interval. Tables II, III, and IV reproduce the coefficients for the cases $n=m, n=1,2, \cdots, 7$ for each interval. All coefficients are given to an accuracy slightly greater than that justificd by the maximal errors. These errors should not be affected by reasonable rounding.

Although a detailed study of the numerical stability of the approximations has not been made, Horner's method for evaluating the polynomials involved was reasonably stable in the few cases checked. Conversion to $J$-fraction form resulted in extreme instability.

Argonne National Laboratory

Argonne, Illinois 60439

1. J. R. Rice, "On the $L_{\infty}$ Walsh arrays for $\mathrm{I}(x)$ and $\operatorname{Erf} c(x)$," Math. Comp., v. 18, 1964 pp. $617-626$. MR 29 2233.

2. John H.art et aL, Handbook of Computer Approximations, Wiley, New York. (To appear.)

3. M. Abramowitz \& I. A. Stegun (Eds.), Handbook of Mathematical Functions, Appl. Math. Series, Vol. 55, National Bureau of Standards, U. S. Government Printing Office, Washington, D. C., 1964.

4. W. J. Cody \& Joseph Stoen, "Rational Chebyshev approximations using interpolation." (To appear.) 